\title{
THE EQUATIONS OF MOTION FOR BINARY SYSTEMS WITH RELATIVISTIC QUADRUPOLE-QUADRUPOLE MOMENTS INTERACTION
}

\author{
C. XU AND X. WU \\ Dept. of Phys., Nanjing Normal Univ., \\ Nanjing 210097, P.R.China
}

The study of binary systems is one of the most important problems in astronomy. Especially recently, gravitational wave detection made possible by Laser Interferometer Gravitational wave Observatory - LIGO and VIRGO, LISA opens up a completely new window for the observation of our universe, it becomes one of the most important and forward area in the modern general relativistic astrophysics. Coalescing binary neutron star (NS) systems are believed to be the most important source emitted high-frequency gravitational wave. Therefore the study of NS coalescence is regarded as a major challenge in modern relativistic astrophysics. Indeed, if the two-body problem could be solved with a sufficient accuracy, the wealth of information might be extracted from the waveforms of coalescing binaries. Many early works to derive and investigate the gravitational two-body system with spin and quadrupole moment interaction have been done already. A detail appraisal of their works has been made by $\mathrm{Xu}, \mathrm{Wu}$ and Schäfer ${ }^{[1]}$ where they derived the first post-Newtonian equations of motion for binary systems with monopole, spin and quadrupole interaction by making use of the scheme developed by Damour, Soffel and Xu (DSX) ${ }^{[2,3]}$ As we know, in the last stages of coalescence in binary system, the distance between two stars is closer, the tidal force is stronger, so the nonspherical size $l$ is larger, $l^{2} / r^{2}$ can reach the level of $v^{2} / c^{2}$, where $\mathrm{r}$ is the distance between two bodies. In this case, the relativistic qudrupole-quadrupole term is of 3-PN order, so one can not neglect the 1-PN contribution of the q-q terms when 3-PN equations of motion (for mass-monopole) are considered. In order to fit the requirement of more accurate solution for binary system, the relativistic q-q terms in the post Newtonian equation of motion have been calculated in this paper. Our work is the first to obtain explicit 1-PN equa- 
tions of motion for binary systems with relativistic quadrupole-quadrupole interaction in terms of only collective coordinates and B-D moments.

Because of complicated calculation, we use Maple Computer Algebra System as an auxiliary medium to save time and guarantee the correctness. According to Eqs. 5.26, 5.27, A7 and A8 of DSX paper II ${ }^{[3]}$, we first calculated $W^{B / A}$ and $W_{a}^{B / A}$, which are the local external potential of body A produced by body B. Substitute the calculated external potential $W^{B / A}$ and $W_{a}^{B / A}$ into Eqs. A14, A15 of DSX paper II, we obtain the tidal moments. Substitute them into Eq. 6.17c of DSX paper II, we finally obtain the equations of motion for binary systems truncated to relaticistic quadrupolequadrupole moments level in the local coordinate system of body A. From the physical meaning we can decompose them into nine coupling parts of different type (monopole-spin-quadrupole moments of body A coupled with monopole-spin-quadrupole moments of body B):

$$
\begin{aligned}
M^{A} A_{a}^{A}= & F_{a}^{A}\left(M^{A} \times M^{B}\right)+F_{a}^{A}\left(M^{A} \times S^{B}\right)+F_{a}^{A}\left(M^{A} \times Q^{B}\right) \\
& +F_{a}^{A}\left(S^{A} \times M^{B}\right)+F_{a}^{A}\left(S^{A} \times S^{B}\right)+F_{a}^{A}\left(S^{A} \times Q^{B}\right) \\
& +F_{a}^{A}\left(Q^{A} \times M^{B}\right)+F_{a}^{A}\left(Q^{A} \times S^{B}\right)+F_{a}^{A}\left(Q^{A} \times Q^{B}\right)
\end{aligned}
$$

Compare our results to the paper by Xu et al. ${ }^{[1]}$, the 1st, 2nd, and 4, 5, 6, 8rd terms are the same, the another three terms enclosed more relativistic q-q terms. Each q-q term is composed of four parts: the first part is $G / r_{A B}^{6}$ $\left(r_{A B}\right.$ is the distance between body $A$ and $B$ ) with a constant coefficient sometimes which include in a ratio of mass $M^{A}$ (body A) to $M^{B}$ (body $\mathrm{B}$ ) or $M^{B}$ to $M^{A}$; the second part is quadrupole $(\mathrm{Q})$ multiplied by quadrupole(Q) which could be $Q^{A} \times Q^{B}$ or $Q^{A} \times Q^{A}$ or $Q^{B} \times Q^{B}$; the third part is a post Newtonian coefficient $v^{2} / c^{2}$ or $u / c^{2}$ where $v$ might be $v_{A}$ (velocity of body $\mathrm{A}), v_{B}$ (velocity of body $\mathrm{B}$ ) or $v_{A B}$ (relative velocity from $\mathrm{B}$ to $\mathrm{A}$ ) and $u$ might be the potential of $M^{A}$ or $M^{B}$; the fouth part is a spatial position tensor $n_{A B}^{a b c \cdots}$ and/or a spatial Levi Civita symbol $\varepsilon_{a b c}$, the indices of which would be summation with the indices of quadrupoles to just make the index at left side (the index of the acceleration). Since the results are very long, we do not have enough room to write down here detail. We will publish in another place.

If we neglect all the relativistic quadrupole-quadrupole terms, our results are total the same as the paper by $\mathrm{Xu}$ et al. ${ }^{[1]}$. Furthermore, the Eq. 1 for binary systems can be easily extended to n-body equations of motion.

\section{References}

1. C. Xu, X. Wu and G. Schäfer, Phys. Rev. D 55, 528 (1997).

2. T. Damour, M. Soffel and C. Xu, Phys. Rev. D 43, 3273 (1991).

3. T. Damour, M. Soffel and C. Xu, Phys. Rev. D 45, 1017 (1992). 